\title{
Determination of Minutiae Scores for Fingerprint Image Applications *
}

\author{
P. Bhowmick, A. Bishnu, B. B. Bhattacharya ’ M. K. Kundu, C. A. Murthy \\ $\left\{\right.$ partha $\_$, bishnu $t$, bhargab, malay, murthy\}@isical.ac.in \\ Indian Statistical Institute, 203 B. T. Road, Calcutta - 700108 \\ and \\ T. Acharya \\ tinku_acharya@ieee.org \\ Elution Technologies, Phoenix, AZ - 85226, USA
}

\begin{abstract}
Many Automatic Fingerprint Identification Systems (AFIS) are based on minutiae matching. Minutiae are the terminations and bifurcations of the ridge lines in a fingerprint image. A fingerprint image that has undergone binarization, followed by thinning, in order to extract the minutiae, contains hundreds of minutiae, all of which are not so vivid and obvious in the original image. Thus, the set of minutiae that are well-defined and more prominent than the rest should have given higher relevance and importance in the process of minutiae matching.

In this work, a method to assign a score value to each of the extracted minutiae is proposed, based on some topographical properties of a minutia. The score associated to a minutia signifies its genuineness and prominence. A minutia with a higher score value should be given higher priority in the matching scheme to yield better results.
\end{abstract}

\section{Introduction}

A fingerprint image $I$ essentially consists of a set of minutiae on the $x-y$ plane. Minutiae are the terminations and bifurcations of ridge lines in a fingerprint image. The ridge lines, appearing in the foreground of the gray-scale topography, are separated by valley lines appearing in the background. In a fingerprint image, there exists a striking duality in the sense that the valley lines also have minutiae (terminations and bifurcations) and flow patterns similar to the ridge lines $[4,5]$. The ridge and valley characteristics, such as ridge and valley flow directions, inter-ridge and inter-valley distances, ridge and valley breaks, etc., are very useful properties that indicate the validity criteria of a minutia detected by any algorithm. These parameters have been used extensively in a number of earlier works. For enhancing a gray-level fingerprint image, orientation of ridges is used for designing a filter by O'Gorman and Nickerson

\footnotetext{
${ }^{*}$ This work is funded by a grant from Intel Corp., USA (PO \#CAC042717000)

${ }^{\dagger}$ Author for correspondence.
}

[11], and, for using directional images by Mehtre et al [10]. In a work by Hung [5], ridge enhancement is done based on ridge directions, and noise removal and pattern purification are performed with the help of both ridge and valley characteristics.

A gray-scale fingerprint image often undergoes binarization, followed by thinning, in the preprocessing stage, in order to extract the minutia points $[2,6]$. During preprocessing, apart from spurs, bridges or loops, several spurious and misleading lines appear in the thinned image because of the noise present in the original gray-scale image. These lines are mere aberrations that often give rise to poor or notso-obvious minutiae, thereby delaying the process of minutiae matching, or reporting a poor fingerprint match. Spurs, bridges, and loops are easily detectable in a less noisy region. In a substantially large noisy part of an image, several criss-crosses may arise that are not always detectable as bridges or loops. There may also exist some minutiae in a noise-free region (apparently, by the naked eye) that are feebly recognizable in the gray-scale image because of erratic gray-value pattern in that locality. As a result, an ambiguity may arise regarding the inclusion or exclusion of a minutia depending on its visual clarity in the original gray-scale image.

In order to circumvent this uncertainty, we propose a methodology of assigning a score value to each minutia, after elimination of spurs, bridges, and loops. Each minutia is assigned a score in the scale $[1,100]$ depending on its topographical characteristics in the skeletonized ternary image (ridge, valley, and background), which in turn, are derived from its visual prominence in the original gray-scale image.

\section{Score-based fingerprint matching}

Let $A$ be the set of minutiae, called data set, existing in the fingerprint database, and $B$ be the query set of minutiae that has to be checked for a match with some element of $A$. The existing matching schemes do not discriminate among the 


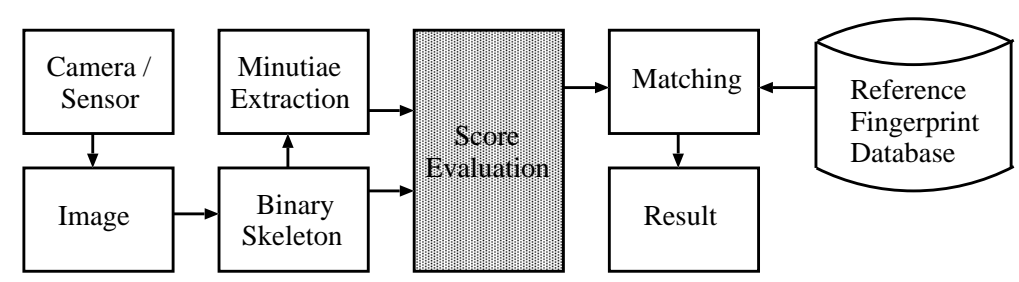

Figure 1: Generic structure of an AFIS with minutiae scores.

minutiae apropos their quality either in the data set or in the query set. A match is reported if the coordinates, types and angles of minutiae of query set $B$ are found to be agreeing with those of data set $A$ under certain transformations like translation, rotation, or scaling $[4,6,7,8,12]$.

In order to consider the relative quality of a minutia in a fingerprint image as a practical matching criterion, we define a minutia point $P$ as a 5-tuple, $P=\langle x, y, t, \theta, s\rangle$, where, $(x, y)=$ coordinates of $P, t=$ type of minutia (a bifurcation minutia or a termination minutia as considered by the Federal Bureau of Investigation and adopted in most AFIS), $\theta=$ angle made by the tangent to the corresponding ridge at the point $(x, y)$, and $s=$ an integer score associated with the minutia $P$.

The score values are normalized within a scale of 1 to 100 , where, a minutia with score nearing 100 is of the highest significance compared to any other minutia with a lower score value. In other words, if a minutia $P_{1}$ has a score $s_{1}$, and another minutia $P_{2}$ has a score $s_{2}$, where $s_{1}<s_{2}$, then $P_{1}$ is a less dependable minutia than $P_{2}$.

While applying a matching procedure based on fingerprint minutiae, the scores of minutiae of $A$ and those of $B$ can be used to tell about how good or bad the match is. If a minutia $\left(x_{a_{i}}, y_{a_{i}}\right)$ with score $s_{a_{i}}$ in set $A$ is a potential match with a minutia $\left(x_{b_{i}}, y_{b_{i}}\right)$ with score $s_{b_{i}}$ in set $B$, the difference $s_{a_{i}} \sim s_{b_{i}}$ indicates the quality of matching of $\left(x_{a_{i}}, y_{a_{i}}\right)$ and $\left(x_{b_{i}}, y_{b_{i}}\right)$. For a matching between $A$ and $B$ with $n$ minutiae, $n \geq 3$, we define the matching index $M I$ as follows:

$$
M I=100-\frac{1}{n} \sum_{\substack{\left(x_{a_{i}}, y_{a_{i}}\right) \in A \\\left(x_{b_{i}}, y_{b_{i}}\right) \in B}}\left|s_{a_{i}}-s_{b_{i}}\right|
$$

Since $1 \leq s_{a_{i}} \leq 100$ and $1 \leq s_{b_{i}} \leq 100$ for $1 \leq i \leq n$, so $0 \leq\left|s_{a_{i}}-s_{b_{i}}\right| \leq 100$, and therefore, $M I$ also lies in the range $[0,100]$. A high value of $M I$ implies a strong match between $A$ and $B$, whereas, a low value indicates a poor one.

The concept of score can be also exploited to expedite the matching procedure between a query set $B$ and a data set $A$. The problem is to check for a matching in $A$, if at all exists, in the fingerprint image database, with respect to the query set $B$. In that case, a small subset $B_{1}$ of minutiae with leading score values in the query set $B$ should be considered first to check for a match with the data set $A$. If the match between $A$ and $B_{1}$ is satisfactory, a next level match can be tried between $A$ and a larger subset of $B$. This may be continued till there is a total match between $A$ and $B$. At any intermediate matching stage involving, say, $A$ and $B_{1}$, if the match is not satisfactory, the remaining set of minutiae, i.e., $B-B_{1}$ need not be tried for, thus saving the matching time for an unsuccessful case. A score-based generic structure of an AFIS is shown in Fig. 1.

\section{Evaluation of score}

The score $s$ of a minutia $P$ is estimated based on the following properties:

- pattern of ridge flow in and around $P$;

- pattern of valley flow in and around $P$;

- noise level in the locality of $P$.

If the ridge and valley lines in the local neighbourhood of $P$ have a smooth nature of flow, the corresponding minutia $P$ will have a genuine contribution in the fingerprint matching. On the contrary, if in some region, the ridge and valley lines have an erratic or uneven nature of flow, a minutia $P^{\prime}$ in that region should not predominate the matching procedure. The former minutia $(P)$, being located in a tidy region, contributes more confidence in the matching procedure than the latter $\left(P^{\prime}\right)$ which is located in a noisy region.

For a minutia $P(x, y)$, the score is given by the equation

$$
s=s_{r i}+s_{v a}+s_{n o}
$$

where, $s_{r i}, s_{v a}$ and $s_{n o}$ are the score components due to ridge flow, valley flow, and noise level respectively in the local neighborhood of $P$. The components $s_{r i}$ and $s_{v a}$ denote measures of perfectness of ridge and valley flow respectively, that are evaluated based on some distances estimated in the local ridge and valley topography around the minutia $P$. To take into account the noise of the region in and around $P$, the component $s_{n o}$ is estimated in a local window centered at $P$. Noise imparts a negative effect on the score.

\subsection{Score of a bifurcation minutia}

Let $\lambda$ be the average inter-ridge distance of a fingerprint image. First, we find the three neighbor pixels $N_{1}, N_{2}, N_{3}$ of $P$, considering 8-neighborhood. $N_{1}, N_{2}, N_{3}$ are the three starting pixels of the ridges $r_{1}, r_{2}, r_{3}$ respectively, incident at $P$. We explore a walk along each of $r_{1}, r_{2}, r_{3}$ starting from $N_{1}, N_{2}, N_{3}$ respectively, each walk being of length $\lambda$. 
Let these walks be named as $w_{1}, w_{2}$, and $w_{3}$ respectively. If during some walk $w_{i}, 1 \leq i \leq 3$, any bifurcation or termination minutia is encountered, the walk is halted. Let, $l_{i}, 1 \leq i \leq 3$, denote the length of the walk $w_{i}$. Let, $l_{\text {min }}$ be the minimum of $l_{i}, 1 \leq i \leq 3$, and $\mu$ be the number of walks whose lengths are less than $\lambda$. If $P$ is a minutia of good quality, then each $l_{i}$ should be at least $\lambda / 2$, and at least two of them should be $\lambda$. So, if $l_{\min }<\lambda / 2$ or, $\mu \geq$ 2 , we assign 0 to score and return from this point. Else, if $l_{\min }<\lambda$, then we walk for a length $l_{\min }$ along each of the three ridges $r_{1}, r_{2}, r_{3}$ starting from $N_{1}, N_{2}, N_{3}$ respectively, so that after the (re-)walks, each of the points $Q_{1}, Q_{2}, Q_{3}$, reached on the three ridges $r_{1}, r_{2}, r_{3}$ respectively, is at equal distance from $P$ (Fig. 2).

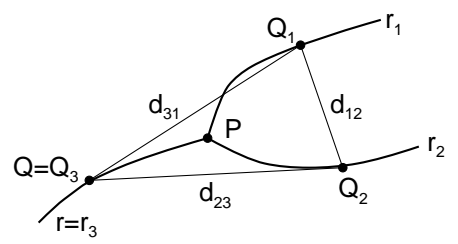

Figure 2: Ridges incident at the bifurcation minutia $P$.

In this scenario, we need to identify the ridge line that bifurcates at $P$. In Fig. 2, the three ridges are shown as $r_{1}, r_{2}$, and $r$, where, w.l.g., $r\left(=r_{3}\right)$ has been depicted as the pre-bifurcated ridge, and $r_{1}, r_{2}$ are its two bifurcations at $P$. To identify the pre-bifurcated ridge, we define $d_{\min }=\min \left(d_{12}, d_{23}, d_{31}\right)$, where, $d_{i j}=L_{2}$-distance between $Q_{i}$ and $Q_{j}, 1 \leq i, j \leq 3, i \neq j$. If $Q_{1}$ and $Q_{2}$ are on the two bifurcated ridges $r_{1}$ and $r_{2}$, then $d_{12}<d_{23}$ and $d_{12}<d_{31}$. However, this condition may fail if $P$ is a poor minutia candidate, viz., when the ridges incident at $P$ are of uneven nature, and it is difficult to ascertain the pre-bifurcated ridge among $r_{1}, r_{2}, r_{3}$. Hence, if $d_{\min }>3 l_{\min } / 2$, we assign 0 to score, and return.

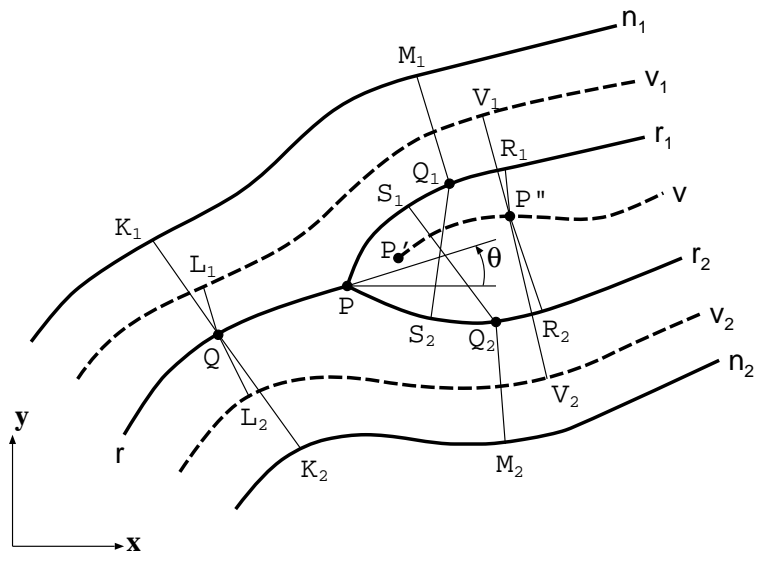

Figure 3: Ridge and valley characteristics around a bifurcation minutia.

In order to compute the score $s_{r i}$ for a bifurcation minu- tia $P$, we define the following distances, vide Fig. 3 . $d_{q n_{1}}=$ distance from $Q$ to neighbor ridge $n_{1}=Q K_{1}$; $d_{q n_{2}}=$ distance from $Q$ to neighbor ridge $n_{2}=Q K_{2}$; $d_{q_{1} n_{1}}=$ distance from $Q_{1}$ to neighbor ridge $n_{1}=Q_{1} M_{1}$; $d_{q_{2} n_{2}}=$ distance from $Q_{2}$ to neighbor ridge $n_{2}=Q_{2} M_{2}$; $d_{q_{1} r_{2}}=$ distance from $Q_{1}$ to bifurcated ridge $r_{2}=Q_{1} S_{2}$; $d_{q_{2} r_{1}}=$ distance from $Q_{2}$ to bifurcated ridge $r_{1}=Q_{2} S_{1}$;

For a good minutia, the above distances should be close to $\lambda$. So, $s_{r i}$ is assigned to $P$ depending on the closeness of $d_{B M, r i} \in\left\{d_{q n_{1}}, d_{q n_{2}}, d_{q_{1} n_{1}}, d_{q_{2} n_{2}}, d_{q_{1} r_{2}}, d_{q_{2} r_{1}}\right\}$ w.r.t. $\lambda$. Thus, for a bifurcation minutia $P$, the score w.r.t. the ridge characteristics can be chosen as:

$$
s_{r i}=\alpha_{r i} \sum_{d_{B M, r i}}\left(\lambda-\left|\lambda-d_{B M, r i}\right|\right)
$$

where, $\alpha_{r i}$ is the ridge score multiplier for bifurcation minutiae.

Similarly, the score $s_{v a}$ for the bifurcation minutia $P$ is based on the following set of distances.

$d_{q v_{1}}=$ distance from $Q$ to neighbor valley $v_{1}=Q L_{1}$;

$d_{q v_{2}}=$ distance from $Q$ to neighbor valley $v_{2}=Q L_{2}$;

$d_{p p^{\prime}}=$ distance from $P$ to valley termination minutia $P^{\prime}$, if any, lying near $P$ in between $r_{1}$ and $r_{2}=P P^{\prime}$;

$d_{p^{\prime \prime} r_{1}}=$ distance from $P^{\prime \prime}$ to bifurcated ridge $r_{1}=P^{\prime \prime} R_{1}$;

$d_{p^{\prime \prime} r_{2}}=$ distance from $P^{\prime \prime}$ to bifurcated ridge $r_{2}=P^{\prime \prime} R_{2}$;

$d_{p^{\prime \prime} v_{1}}=$ distance from $P^{\prime \prime}$ to neighbor valley $v_{1}=P^{\prime \prime} V_{1}$;

$d_{p^{\prime \prime} v_{2}}=$ distance from $P^{\prime \prime}$ to neighbor valley $v_{2}=P^{\prime \prime} V_{2}$;

where, $P^{\prime \prime}$ is the point along the valley $v$ at a distance $\lambda$ from $P^{\prime}$, or, a bifurcation or termination of $v$ appearing within the target walk-length of $\lambda$.

While the parameter $\left\{d_{B M, r i}\right\}$ represents some kind of inter-ridge distance, we define other distance measures with a subtle difference. Distances in the set $\left\{d_{B M, v a}^{1}\right\}$ $=\left\{d_{p^{\prime \prime} v_{1}}, d_{p^{\prime \prime} v_{2}}\right\}$ are inter-valley distances, which should be ideally close to $\lambda$. The other set $\left\{d_{B M, v a}^{2}\right\}=$ $\left\{d_{q v_{1}}, d_{q v_{2}}, d_{p p^{\prime}}, d_{p^{\prime \prime} r_{1}}, d_{p^{\prime \prime} r_{2}}\right\}$ contains distances from a ridge point to a valley line, or from a valley point to a ridge line, and therefore, requires a flexibility in their contribution to $s_{v a}$. Hence, distances in the set $\left\{d_{B M, v a}^{1}\right\}$ are very much similar to $\left\{d_{B M, r i}\right\}$ as far as the estimation of $s_{v a}$ is concerned. Their contribution to score may be chosen as:

$$
s_{v a}^{1}=\alpha_{v a} \sum_{d_{B M, v a}^{1}}\left(\lambda-\left|\lambda-d_{B M, v a}^{1}\right|\right) ;
$$

And, that due to $\left\{d_{B M, v a}^{2}\right\}$ is

$$
s_{v a}^{2}=\sum_{d_{B M, v a}^{2}} s_{d_{B M, v a}^{2}}
$$


where, $s_{d_{B M, v a}^{2}}$ is chosen as:

$s_{d_{B M, v a}^{2}}=\left\{\begin{array}{lll}\alpha_{v a} \lambda & \text { if } & \lambda / 4 \leq d_{B M, v a}^{2} \leq 3 \lambda / 4 \\ \alpha_{v a}\left(d_{B M, v a}^{2}-\lambda / 4\right) & \text { if } & d_{B M, v a}^{2}<\lambda / 4 \\ \alpha_{v a}\left(3 \lambda / 4-d_{B M, v a}^{2}\right) & \text { if } & d_{B M, v a}^{2}>3 \lambda / 4\end{array}\right.$

and $\alpha_{v a}$ is the valley score multiplier for a bifurcation minutia.

\subsection{Score of a termination minutia}

Let $P$ be a termination minutia and $N$ be the adjacent ridge pixel of $P$, considering 8-neighborhood. Since $P$ is a termination minutia, there will be only one ridge line, say $r$, incident at $P$ [Fig. 4]. We walk along $r$ starting from $N$, for a length $\lambda$, and designate the walk as $w$. Let $l$ denote the length of the walk. Since a skeletonized fingerprint image should be devoid of spurs and bridges, $l$ should always be equal to $\lambda$. Let $Q$ be the point on the ridge $r$ reached

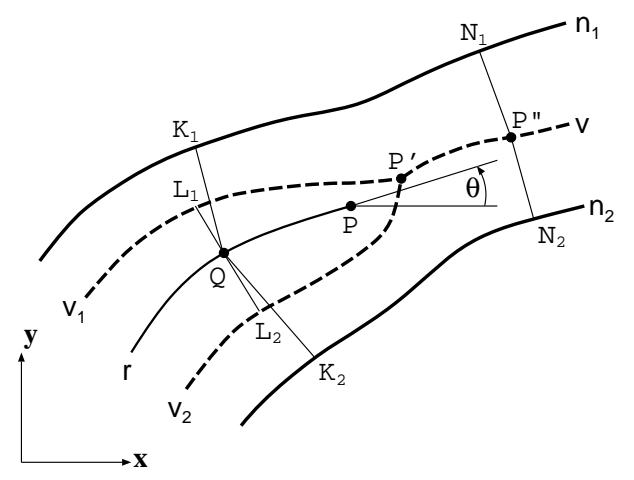

Figure 4: Ridge and valley characteristics around a termination minutia.

after the walk $w$. For estimation of the score $s_{r i}$ for the termination minutia with respect to ridge lines in the region containing $P$, we define the set $\left\{d_{T M}\right.$,ri $\}$ of following distances.

$d_{q n_{1}}=$ distance from $Q$ to neighbor ridge $n_{1}=Q K_{1}$;

$d_{q n_{2}}=$ distance from $Q$ to neighbor ridge $n_{2}=Q K_{2}$;

For $P$ to be a termination minutia of good quality, the above distances, should be close to $\lambda$. These distances are basically inter-ridge distances similar to $\left\{d_{B M, r i}\right\}$ in the case of bifurcation minutiae. Hence, the score $s_{r i}$ is assigned to $P$ based on the following equation that resembles with Eqn. 2 in form:

$$
s_{r i}=\beta_{r i} \sum_{d_{T M, r i}}\left(\lambda-\left|\lambda-d_{T M, r i}\right|\right)
$$

where, $\beta_{r i}$ is the ridge score multiplier for termination minutiae.

Similarly, the score $s_{v a}$ for the termination minutia $P$ is based on the set $\left\{d_{T M, v a}\right\}$ of following distances. $d_{q v_{1}}=$ distance from $Q$ to neighbor valley $v_{1}=Q L_{1}$; $d_{q v_{2}}=$ distance from $Q$ to neighbor valley $v_{2}=Q L_{2}$;

$d_{p p^{\prime}}=$ distance from $P$ to valley termination minutia $P^{\prime}$, if

any, lying near $P$ in between $n_{1}$ and $n_{2}=P P^{\prime}$;

$d_{p^{\prime \prime} n_{1}}=$ distance from $P^{\prime \prime}$ to neighbor ridge $n_{1}=P^{\prime \prime} N_{1}$;

$d_{p^{\prime \prime} n_{2}}=$ distance from $P^{\prime \prime}$ to neighbor ridge $n_{2}=P^{\prime \prime} N_{2}$;

where, $P^{\prime \prime}$ is the point along the valley $v$ at a distance $\lambda$ from $P^{\prime}$, or, a bifurcation or termination of $v$ appearing within the target walk-length of $\lambda$.

The above set of distances are measured either from a ridge point to a valley line or from a valley point to a ridge line. Hence, their contribution to score $s_{v a}$ is given by:

$$
s_{v a}=\sum_{d_{T M, v a}} s_{d_{T M, v a}}
$$

where, $s_{d_{T M, v a}}$ is chosen as:

$s_{d_{T M, v a}}=\left\{\begin{array}{lll}\beta_{v a} \lambda & \text { if } & \lambda / 4 \leq d_{T M, v a} \leq 3 \lambda / 4 \\ \beta_{v a}\left(d_{T M, v a}-\lambda / 4\right) & \text { if } & d_{T M, v a}<\lambda / 4 \\ \beta_{v a}\left(3 \lambda / 4-d_{T M, v a}\right) & \text { if } & d_{T M, v a}>3 \lambda / 4\end{array}\right.$

and $\beta_{v a}$ is the valley score multiplier for a termination minutia.

\subsection{Estimation of noise}

Let $P$ be a bifurcation or termination minutia having a positive score after the evaluation of $s_{r i}$ and $s_{v a}$. If $P$ does not have a positive score, we need not evaluate $s_{n o}$, since $s_{n o}$ will contribute a negative score to $P$; finally we will consider only the set of minutiae with postive scores. Consider a circular window $W$ of radius $R=N \lambda$ around $P(x, y)$, vide Fig. 5. Let $\left\{Q_{i} \mid Q_{i}\right.$ lies within $\left.W ; i=1,2, \ldots, \eta\right\}$ be the set of points, with each point $Q_{i}$ satisfying any one of the following 3 properties (Fig. 5):

(i) $Q_{i}$ is a ridge minutia with $s_{r i}+s_{v a}=0$;

(ii) $Q_{i}$ is a non-minutia ridge point having three or more ridges incident upon it;

(iii) $Q_{i}$ is either a valley bifurcation or a valley termination minutia.

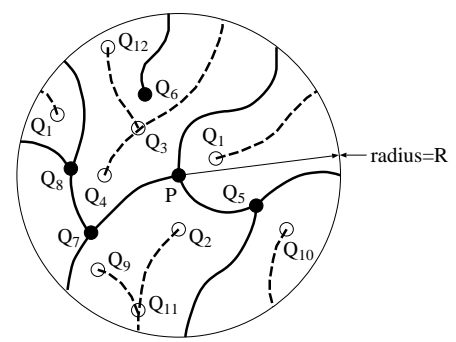

Figure 5: Contributing points $\left\{Q_{1}, Q_{2}, \ldots, Q_{12}\right\}$ in a noisy window $W$ centered around the minutia $P$.

The above definition enables us to use $\left|\left\{Q_{i}\right\}\right|=\eta$ as a measure of noise level in the window $W$ centered around $P$. We define another parameter $\nu$, called the noise factor, 
which is used to find the noise threshold $\tau_{\text {noise }}$ given in the equation below, that will indicate whether or not a window $W$ associated with a minutia $P$ is noisy:

$$
\tau_{n o i s e}=\nu N
$$

If $\eta$ is higher than $\tau_{\text {noise }}$ in $W$ corresponding to $P$, the noise level in $W$ is considered high enough and each point $Q_{i}, i=1,2, \ldots, \eta$, is accounted one by one for their individual contribution to the noise-induced (negative) score $s_{n o}$ of $P$. Thus, Eqn. 10 can be used to find $s_{n o}^{i}$ attributed by each $Q_{i}$, and Eqn. 11 sums up the individual scores to compute the total score due to noise.

$$
\begin{gathered}
s_{n o}^{i}=\gamma\left(R-L_{2}\left(P, Q_{i}\right)\right) \\
s_{n o}=\left\{\begin{array}{lll}
0 & \text { if } & \eta \leq \tau_{n o i s e} \\
\sum_{i=1}^{\eta} s_{n o}^{i} & \text { if } & \eta>\tau_{n o i s e}
\end{array}\right.
\end{gathered}
$$

where, $\gamma$ is the noise score multiplier.

In Eqn. $10, L_{2}$-distance between two points $P_{1}\left(x_{1}, y_{1}\right)$ and $P_{2}\left(x_{2}, y_{2}\right)$ is given by:

$$
L_{2}\left(\left(x_{1}, y_{1}\right),\left(x_{2}, y_{2}\right)\right)=\sqrt{\left\{\left(x_{1}-y_{1}\right)^{2}+\left(x_{2}-y_{2}\right)^{2}\right\}}
$$

\section{Experiments and results}

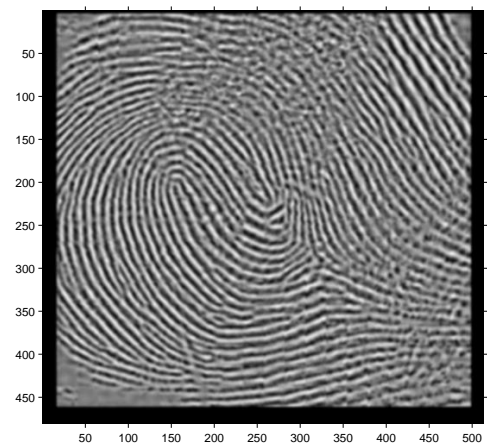

Figure 6: A sample fingerprint image from NIST $14 \mathrm{sdb}$.

We used several fingerprint images from the NIST Special Database 14 [1] and NIST Special Database 4 [13]. In order to keep the minutiae scores of the order of 100 prior to normalization, the value of $\alpha_{r i}\left(=\alpha_{v a}\right)$ has been chosen as 1.00 .

For evaluating the score of a bifurcation minutia, we need to compute 6 distances in the set $\left\{d_{B M, r i}\right\}$, measured w.r.t. different ridge lines, and 7 distances in the set $\left\{d_{B M, r i}\right\}$, measured w.r.t. different valley lines. For finding the score of a termination minutia, we need 2 and 3 such distances, in the sets $\left\{d_{T M, r_{i}}\right\}$ and $\left\{d_{T M, v a}\right\}$, respectively. Thus, in order to have parity in the score values of bifurcation and termination minutiae, we choose $\beta_{r i}=(6 / 2) \alpha_{r i}=$ 3.0 and $\beta_{v a}=(7 / 3) \alpha_{v a}=2.33$.
Table 1: Score Values of Minutiae

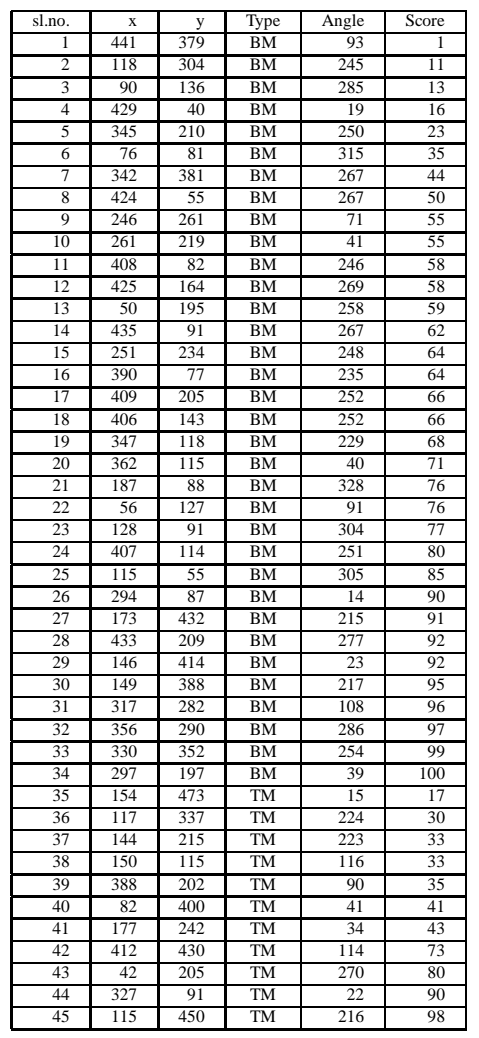

In the estimation of noise-based score, $\nu$ is a controlling paramater that decides the effect of noise on the score. From Eqn. 9, it is evident that a higher value of $\nu$ will enforce a lesser impact of noise in the score. On the basis of our experimental results, we have emperically chosen $N=2, \nu$ $=3$, and, $\gamma=1 / \nu=0.33$.

In Fig. 6, a sample fingerprint image of size $480 \times 512$ is shown. The corresponding ternary skeleton image is shown in Fig. 7, where the darker lines represent the ridges and the faint lines are valleys. The minutiae having positive scores are shown in Fig. 7, with the darkness of a minutia being proportional to its score. Table 1 includes the scores (positive values only) of the bifurcation minutiae (BM), followed by those of the termination minutiae (TM), arranged in ascending orders. The bifurcation minutia at $(297,197)$ has the maximum score 100 , which is well justified by its visual clarity in the image shown in Fig. 6 and the topographical orderliness in its neighborhood in Fig. 7. On the other hand, the minutia at $(441,379)$ is located in a highly noiseaffected region. Scores of some minutiae are written beside the corresponding minutiae in Fig. 7.

The proposed method is implemented in $\mathrm{C}$ on a Sun_Ultra 5_10, Sparc, $233 \mathrm{MHz}$, the OS being the SunOS Release 5.7 Generic. The total CPU time for the evaluation of scores of all minutiae in a ternary skeletonized fingerprint image was found to be around 0.03 to $0.07 \mathrm{sec}$. 


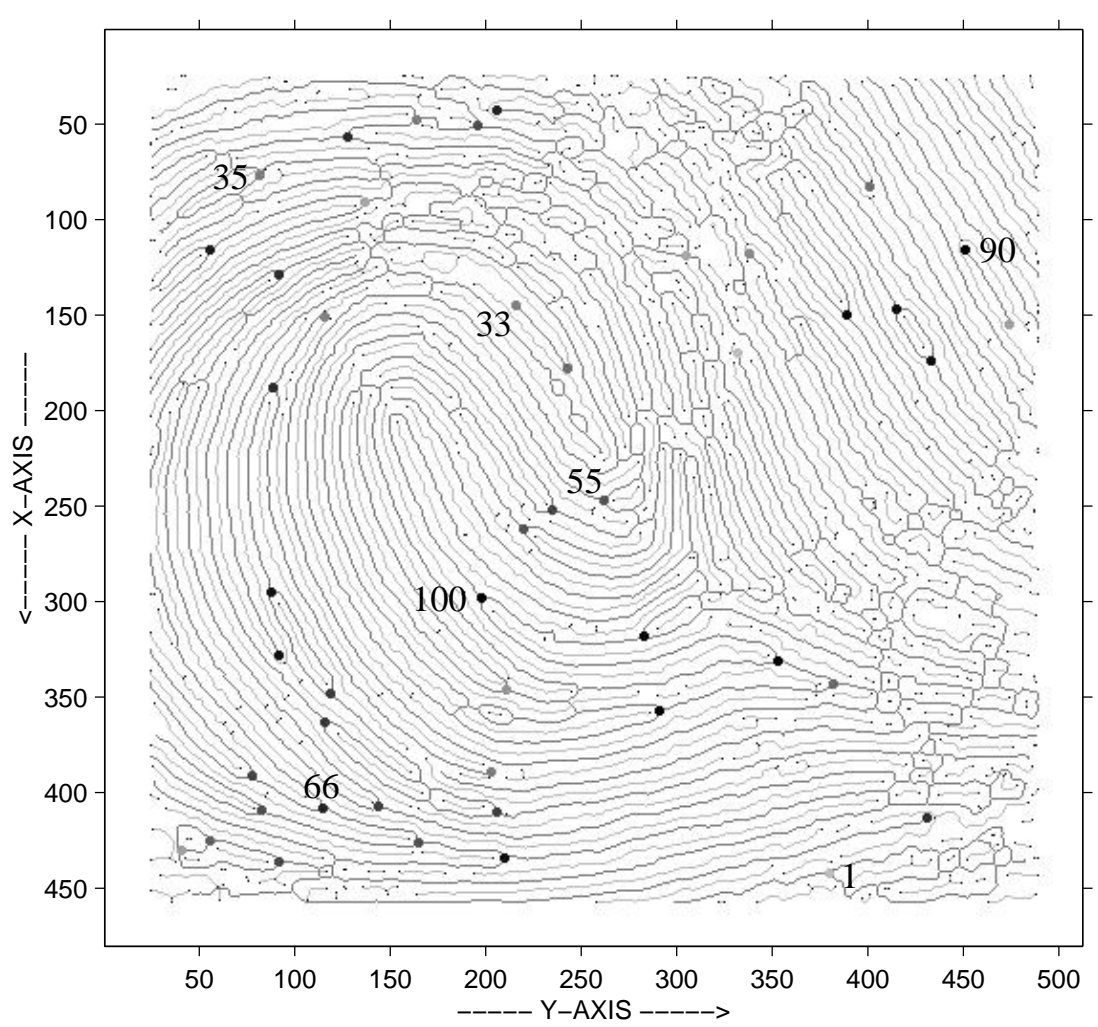

Figure 7: Minutiae shown with darkness proportional to scores.

\section{Conclusions and future works}

A method of scaling to assess a minutia for fingerprint matching is reported in this paper. Development of a faster and realistic fingerprint matching technique based on the proposed method is currently in progress. Some of the empirical formulae mentioned in this paper may require further refinements for more accurate matching result. In reality, the score of a minutia in a query image may be drastically different from that of the database image. If the scores vary widely, then the confidence in matching may reduce significantly. These anomalies have to be resolved to ensure a matching result.

\section{References}

[1] G. T. Candela, P. J. Grother, C. I. Watson, R. A. Wilkinson, and C. L. Wilson. PCASYS - A Pattern-Level Classification Automation System for Fingerprints, NISTIR 5647. National Institute of Standards and Technology, August 1995.

[2] A. Farina, Zs. M. Kovács-Vajna, and A. Leone. Fingerprint Minutiae Extraction from skeletonized binary images. Pattern Recognition, vol. 32, pages 877-889, 1999.

[3] R. Haralick. Ridges and Valleys on Digital Images. Comput. Vis. Graph. Imag. Process., vol. 22, pages 28-38, 1983.

[4] A. K. Hrechak and J. McHugh. Automated Fingerprint Recognition Using Structural Matching. Pattern Recognition, vol. 23, pages 893-904, 1990.
[5] D. C. D. Hung. Enhancement and Feature Purification of Fingerprint Images. Pattern Recognition, vol. 26, pages 16611671, 1993.

[6] A. Jain, L. Hong, and R. Bolle. On-Line Fingerprint Verification. IEEE Transactions on Pattern Analysis and Machine Intelligence, vol. 19, pages 302-313, 1997.

[7] Zs. M. Kovács-Vajna. A Fingerprint Verification System Based on Triangular Matching and Dynamic Time Warping. IEEE Transactions on Pattern Analysis and Machine Intelligence, vol. 22, pages 1266-1276, 2000.

[8] D. Maio and D. Maltoni. Direct Gray-Scale Minutiae Detection In Fingerprints. IEEE Transactions on Pattern Analysis and Machine Intelligence, vol. 19, pages 27-39, 1997.

[9] B. M. Mehtre and N. N. Murthy. A Minutia Based Fingerprint Identification System. in Proceedings Second International Conference on Advances in Pattern Recognition and Digital Techniques, Calcutta 1986.

[10] B. M. Mehtre, N. N. Murthy, S. Kapoor, and B. Chatterjee. Segmentation of Fingerprint Images Using Directional Image. Pattern Recognition, vol. 20, pages 429-435, 1987.

[11] L. O'Gorman and J. V. Nickerson. An Approach to Fingerprint Filter Design. Pattern Recognition, vol. 22, pages 2938, 1989.

[12] F. Pernus, S. Kovacic, and L. Gyergyek. Minutiae-Based Fingerprint Recognition. in Proc. Fifth International Conference on Pattern Recognition, pages 1380-1382, 1980.

[13] C. I. Watson and C. L. Wilson. Fingerprint Database. National Institute of Standards and Technology. Special Database 4, FPDB, April, 1992. 